\title{
Autorenindex/Author Index
}

Backhaus, S. 236

Bals-Pratsch, M. 186

Bauerfeind, I. 70

Berger, A. 41

Bitterlich, N. 61

Burstein, C. 278

Calatzis, A. 239

Cullen, P. 193

Deufel, T. 93

Eberl, W. 248

Fingerle, V. 141

Fraunberger, P. 294

Fuchs, T. 206

Funke, H. 193

Gaida, B. 149

Grammer, T. 109

Haeckel, R. 87

Hafner, G. 218

Halbmayer, W.-M. 56

Haushofer, A.C. 56

Hehr, A. 186

Hehr, U. 186

Heinemann, V. 70

Heinrich, U. 171

Hofmann, D. 76

Hornung, H.-M. 76

Jäger, L. 206

Jarisch, R. 214

Kahlert, S. 70

Kessler, H.H. 56

Kiehntopf, M. 93

Klein, H.-G. 171, 193

Kleine-Tebbe, J. 206

Knabbe, C. 193

Knippel, A.-J. 161

Kohse, K.P. 21
Kostner, G.M. 109

Kozlowski, P. 161

Laessig, D. 70

Lang, T. 254

Langmann, T. 193

Lapin, A. 2

Lau-Werner, U. 76

Lepp, U. 206

Liebenthal, C. 149

Luthe, H. 236

Macdonald, R. 90

März, W. 109, 125, 135

Meinitzer, A. 125

Merten, U.P. 196

Merz, M. 262

Montag, B. 236

Müller, U. 214

Nagel, D. 70,76

Nauck, M. 1

Neumaier, M. 193

Niggemann, B. 206

Patzke, J. 262

Pfeiffer, A. 226

Pilz, S. 135

Plecko, T. 226

Przybilla, B. 214

Rabenau, H.F. 41, 48

Rost, I. 171

Ruëff, F. 214

Rupprecht, W. 171

Saloga, J. 206

Schalhorn, A. 76

Scharnagl, H. 109

Scheithauer, M.-O. 201

Schiessl, B. 171

Schmidt, F.-P. 149

Schneider, J. 61
Seifert, B. 186

Siegemund, A. 262

Siegmund, R. 93

Siekmeier, R. 109

Soltau, U. 218

Spitzenberger, F. 218

Stachon, A. 8

Steiner, M. 1

Steinhorst, A. 41, 48

Stelzl, E. 56

Stenman, U.H. 76

Stieber, P. 70, 76

Stojakovic, T. 109

Stressig, R. 161

Tumani, H. 201

Untch, M. 70

Vieluf, I. 206

Vieths, S. 206

Vinks, A.A. 24

Volk, H.-D. 149

von Ahsen, N. 278

von Baehr, R. 149

von Baehr, V. 149

von Depka, M. 254

Wagner, A. 171

Walli, A.K. 294

Walson, P.D. 24

Weissel, M. 15

Wencker, M. 39

Werfel, T. 206

Wichmann, M.W. 76

Wick, M. 201

Wieland, E. 226

Wilske, B. 141

Wosniok, W. 87

Zimmermann, B.G. 165

Zuberbier, T. 206 